\title{
Echocardiography parameters before therapy cannot predict cardiotoxicity caused by Trastuzumab
}

\author{
Ivo Darko Gabrić*, Ljubica Vazdar, Danijel Planinc, Ozren Vinter, Matias Trbušić, Nikola Bulj, \\ Hrvoje Pintarić \\ University Hospital Center "Sestre milosrdnice", Zagreb, Croatia
}

Cardiotoxicity is the most important side effect of trastuzumab, humanized monoclonal antibody to the HER2 protein, in use for immunotherapy of breast cancer. Cardiotoxicity is mainly manifested as a reduction in left ventricular contractility without myocardial necrosis, and the process is therefore mostly reversible. However, sometimes the disease can progress to irreversible dilated cardiomyopathy. Transthoracic echocardiography is the primary diagnostic method for the assessment of cardiotoxicity and immunotherapy is canceled or suspended if the left ventricular ejection fraction (LVEF) is reduced by $15-16 \%$ from the baseline or to $10-$ $15 \%$ of normal values.

In our study, 130 patients (pts) with non-metastatic breast cancer were treated for one year, in adjuvant therapy, with trastuzumab. According to current guidelines echocardiography was performed before the beginning and in three months period during immunotherapy with trastuzumab. Patients with proven cardiotoxicity were suspended from the therapy for one month and a control echocardiography was performed. Patients were divided in two groups: 51 pts with proven cardiotoxic side effect were assorted into group $A$, and the control group B with 79 pts who didn't have cardiotoxicity.

There were no difference between the groups at the baseline echocardiography in LVEF between the two groups (A : $B=64.49 \pm 4.9 \%: 63.58 \pm 4.3 \%, p=0.6151)$, end-diastolic size of the $\operatorname{LV}(A: B=48.39 \pm 4.1: 47.11 \pm 4.7, p=0.1178)$, wall thickness $(A: B=10.02 \pm 1.54: 9.88 \pm 1.89 \mathrm{~mm}, \mathrm{p}=$ 0.8109 ) and diastolic function.

In patients with reported cardiotoxic effects of trastuzumab (group A) medium lowest LVEF were $44.18 \% \pm 9.9 \%$. After stopping trastuzumab for one month the control LVEF also remained significantly lower in comparison with the control group $B(A: B=53 \pm 8.2 \%: 61.6 \pm 3.7 \%, p=0.0000)$. Entirely reversible cardiac damage was observed in 28 patients $(54.9 \%)$ and irreversible or partially reversible in 23 patients (45.1\%).

Although in several studies initial lower LVEF were found to be a risk factor for the development of cardiotoxicity, we did not determine that any of the echocardiography parameters prior to the trastuzumab therapy was a significant risk factor for the development of cardiotoxicity.

KEYWORDS: cardiotoxicity, trastuzumab, echocardiography, breast cancer.

\section{Received: $20^{\text {th }}$ Mar 2013}

*Address for correspondence: Klinički bolnički centar "Sestre milosrdnice", Vinogradska cesta 29, HR-10000 Zagreb, Croatia.

Phone: +385-1-3787-440

E-mail: idgabric@gmail.com

\section{Literature}

1. Seidman A, Hudis C, Pierri MK, Shak S, Paton V, Ashby M, et al. Cardiac dysfunction in the trastuzumab clinical trials experience. J Clin Oncol. 2002;20(5):1215-21.

2. Fox KF. The evaluation of left ventricular function for patients being considered for, or receiving Trastuzumab (Herceptin) therapy. Br J Cancer. 2006;95(10):1454.

3. Suter TM, Procter M, van Veldhuisen DJ, Muscholl M, Bergh J, Carlomagno C, et al. Trastuzumab-associated cardiac adverse effects in the herceptin adjuvant trial. J Clin Oncol. 2007;25(25):3859-65.

4. Gabrić ID, Pintarić H, Vazdar LJ, Štefanović M, Jazvić M, Soldić Ž, et al. Influence of angiotensin conversion enzyme gene polymorphism on cardiotoxicity caused by immunotherapy with trastuzumab. Circulation. 2012;126;A16961. 